\title{
Machine Learning Based Classification of Myocardial Infarction Conditions Using Smartphone-Derived Seismo- and Gyrocardiography
}

\author{
Saeed Mehrang ${ }^{1}$, Mojtaba Jafari Tadi ${ }^{1}$, Matti Kaisti ${ }^{1}$, Olli Lahdenoja ${ }^{1}$, Tuija Vasankari ${ }^{2}$, Tuomas \\ Kiviniemi $^{2}$, Juhani Airaksinen ${ }^{2}$, Tero Koivisto ${ }^{1}$, Mikko Pänkäälä ${ }^{1}$ \\ ${ }^{1}$ Department of Future Technologies, University of Turku, Finland \\ ${ }^{2}$ Heart Center, Turku University Hospital, Finland
}

\begin{abstract}
In this paper, we attempt to classify the pre- and postoperation cardiac conditions of ST-elevation myocardial infarction (STEMI) utilizing seismocardiography (SCG) and gyrocardiography $(G C G)$ signals recorded solely by a smartphone. SCG and GCG signals were recorded from 20 MI patients who were admitted to Emergency Department of Turku Hospital. Two measurements were recorded from each subject, one before they proceeded to percutaneous coronary intervention (pre-operation) and one afterwards (post-operation) with an average time interval of 2 days. Noise and artefact removal were applied to the signals and subsequently 25 features were extracted. Two classification algorithms, random forest $(R F)$ and support vector machines (SVM), were deployed to discriminate the two cardiac conditions. Accuracy rates of $74 \%$ and $78 \%$ were obtained for RF and SVM, respectively. The results indicate that smartphone SCG-GCG based ischaemia analysis has clinical implications that warrants further investigations.
\end{abstract}

\section{Introduction}

Cardiovascular diseases (CVD) remains to be the most common cause of death in the world [1]. Among CVDs, sudden cardiac death accounts for approximately $50 \%$ of the cardiovascular deaths [1]. Unfortunately, the majority of fatalities in the community occur in low risk coronary patients or in individuals without any history of cardiac diseases [1]. For this group, the challenge is how to help them to interpret the unexpected symptoms correctly and make them to call an ambulance instead of waiting the symptoms to disappear. Advanced therapies like coronary angioplasty or implantable defibrillators are of no value to the majority of victims, who do not survive to reach the medical care.

Acute myocardial infarction (MI) is a condition where the coronary arteries become fully blocked due to accu- mulated plaques. Monitoring cardiac conditions of the MI patients after receiving percutaneous coronary intervention (PCI) and hospital discharge remains to be challenging. It has been shown that, early physician follow-up (within 7 days) has a significant impact on reducing readmission [2]. However, long waiting periods may hinder the intime examination. Early detection of MI after its onset has a great impact on the survival rate and the rehabilitation process of the patients [3]. However, it usually takes a long time until patients are transferred to clinics, diagnosed, and respectively treated [3]. Reducing the diagnosis time requires an easy to follow and accessible examination technique. Remote cardiac monitoring techniques in this case are of significant value [4]. A proper remote monitoring technique is required to be pervasive, available at low cost, and portable. On the other hand, to remotely monitor MI patients who are generally elderly adults, additional hardware to purchase or complex preparations reduce the pervasiveness and success rate. Cardiac monitoring using smartphone mechanocardiography (MCG) sounds appropriate as it requires neither additional devices nor special instructions to follow. Seismocardiography (SCG) [5] and gyrocardiography (GCG) [6] have been considered for cardiac monitoring in a number of different studies and provided promising results [7].

This study aimed to investigate the feasibility of cardiac monitoring of ST-elevation myocardial infarction (STEMI) patients using solely a smartphone. Such a monitoring system can be deployed to assess whether the mechanical functioning of the heart has changed after receiving PCI. The measurement can be carried out quickly and with minimal preparations by a single smartphone placed on the chest without the need for any additional devices.

The contributions of this paper as compared to our previously published results [8] are new improved artefact removal and new features used in classification of pre- and post-operation conditions of STEMI. The artefact removal is crucial in pre-operation measurements and in the intended final use case, because patients may have chest pain 
and anxiety which exacerbates conditions for collection of mechanocardiographic data.

\section{Materials}

The data analyzed in this study comprises of measurements from $20 \mathrm{MI}$ patients who were admitted to the Emergency Department of Turku Hospital. From each patient, one measurement was acquired immediately upon the admission and another one after receiving treatment (PCI).

The lengths of measurements were all less than 3 minutes with a sampling rate of $200 \mathrm{~Hz}$. The data consists of triaxial SCG as well as triaxial GCG signals [6] captured by the inertial measurement unit of a single smartphone placed on the bare chest of the study subjects. The subjects were all in supine position while the measurements were captured.

We considered patients having diagnosed STEMI by a standard examination including 12-lead ECG and biomarker tests. Informed consents were collected from all study subjects and Helsinki declaration was strictly followed. Ethical approval was obtained in advance from the Ethics Committee of Hospital District of the South-West Finland.

\section{Methods}

\subsection{Pre-processing}

A flowchart of all the different steps of analysis pipeline is presented in Fig. 1.

The raw SCG and GCG signals were all bandpass filtered using a $4^{\text {th }}$ order Butterworth filter with pass-band frequencies between 3 to $50 \mathrm{~Hz}$. Baseline drift, respirationinduced chest movements, and high-frequency noise were removed by the aforementioned filtering. To account for the unwanted smartphone movements induced by turning on/off the recording, a period of 2 seconds were dropped from the data at the beginning and end of the recordings.

\subsection{Artefact Removal}

Smartphone acquired signals from the chest were slightly contaminated by movement artefacts. Artefact removal was handled by adopting a new method deploying segmentation and entropy estimation. This process was performed for each of the SCG and GCG signals separately. In detail, the triaxial signal vector magnitude for the SCG and GCG signals were computed and were split into non-overlapping segments of 1 second and for each segment Shannon entropy was estimated. A vector of Shannon entropy values were therefore obtained for each signal vector magnitude where each value corresponded to a single segment. Outliers of the Shannon entropy vectors were

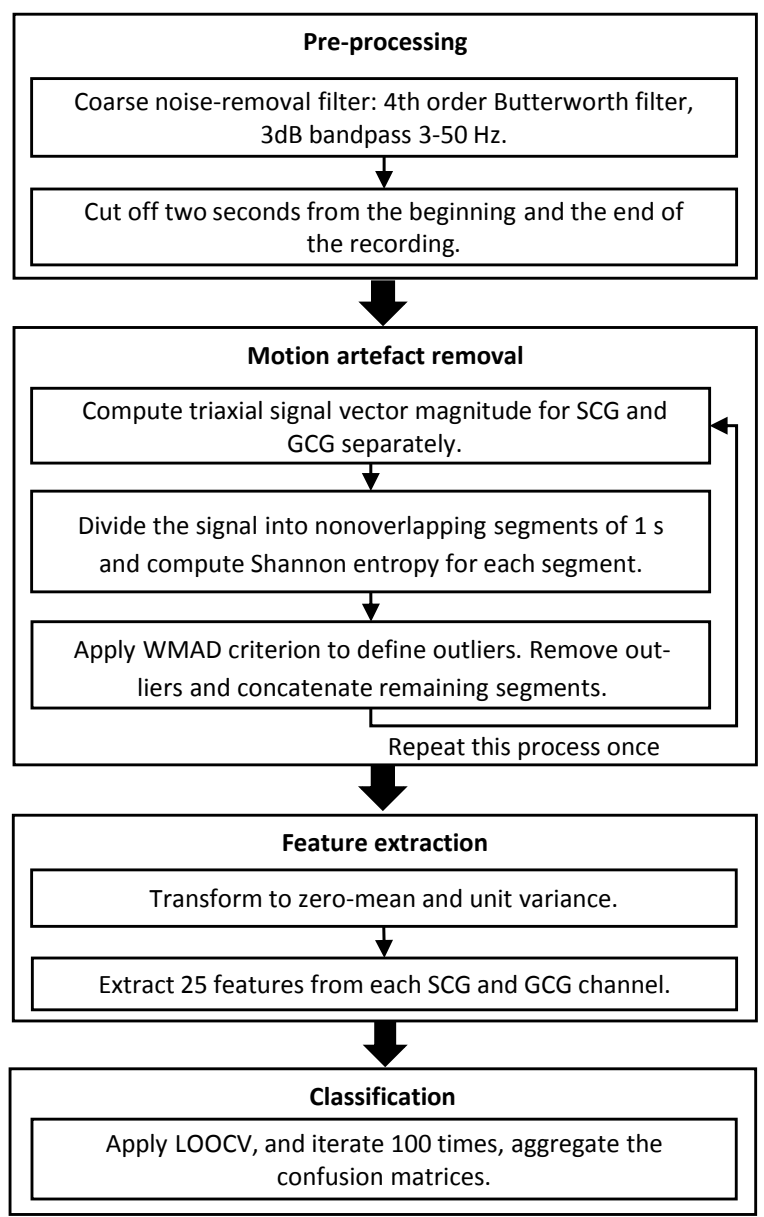

Figure 1. Flowchart of the analysis pipeline.

identified and the segments corresponding to those outlying values were discarded from the original SCG and GCG signals. At the end, the remained segments were concatenated together to reconstruct an artefact-free signal.

Two-stage outlier removal was carried out by first computing Shannon entropy values of the segmented original signals. These Shannon entropy values were then compared against a weighted median absolute deviation (WMAD) criterion. The median absolute deviation [9] was weighted by defining a new variable equal to the fraction of interpercentile range [10-90 percentile] and interquartile range [25-75 percentile]. Signal segments corresponding to the outlying values (artefact segments) of Shannon entropy vector were discarded and the remaining segments were concatenated to form a reconstructed signal. After the first round of reconstruction, there was another artefact removal following the same above-mentioned process. The reconstructed signals after the second stage of artefact removal were fed into a feature extractor algorithm. 


\subsection{Feature Extraction}

After transforming the artefact-free signals to zero-mean and unit variance, a combination of statistical and signal processing features were extracted from all the signals. From each channel of the data 25 features [10] were extracted as shown in Table 1.

Table 1. List of extracted features.

\begin{tabular}{ll} 
Features & \\
\hline \hline mean & standard deviation \\
25th percentile & 75th percentile \\
interquartile range & median \\
peak-to-peak range & skewness \\
kurtosis & root mean square \\
dominant frequency & spectral centroid \\
relative energy of $\mathrm{DWT}_{D 1}$ & cross-correlation \\
relative energy of $\mathrm{DWT}_{D 2}$ & cross-entropy \\
relative energy of $\mathrm{DWT}_{D 3}$ & mutual information \\
relative energy of $\mathrm{DWT}_{A 3}$ & bandwidth \\
Fourier transform entropy & signal entropy \\
relative Fourier energy entropy & \\
relative DWT energy entropy & \\
relative energy of each axis as compared to signal- \\
vector-magnitude
\end{tabular}

\subsection{Classification and Cross-Validation}

Two classifiers, Random forest (RF) [11] and support vector machine (SVM) with radial basis function kernel [12], were deployed in this study. A forest of size 128 trees [13] was used in this study. Model validation was handled by leave-one-subject-out cross-validation (LOOCV). To clarify the variance of the classification for each classifier, we repeated the LOOCV for 100 iterations and aggregated the results. The results of the repeated LOOCVs are presented by confusion matrices with overall accuracy, sensitivity and specificity calculated. Data pre-processing, feature extraction, and classification were all performed by Python 3.6.

\section{Results}

Segments of length 2 seconds were used for entropy estimation. An example Z-axis SCG signal before and after the 2-stage artefact removal is presented in Fig. 2.

Throughout each LOOCV, two measurements were left out at a time as there were two measurements from each subject, one before receiving PCI treatment (preoperation) and one afterwards (post-operation). The predictions of the classifiers were stored and at the end concatenated. This means, the formed confusion matrices of
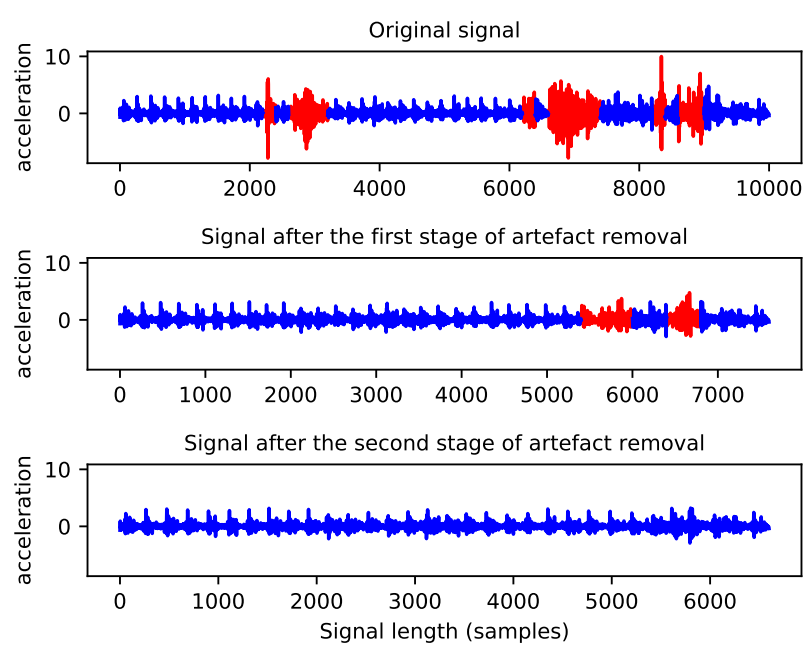

Figure 2. An example of motion-artifact removal from Zaxis of SCG. The top panel shows the band-pass filtered signal. The middle and bottom panels show the outcome of the first and second stages of the artifact removal algorithm. The red color components are selected as motionartifacts to be discarded from the signal.

100 iterations of LOOCV were all aggregated. According to the aggregated confusion matrices, the overall accuracy levels of $74 \%$ and $78 \%$ were obtained for RF and SVM classifiers. For RF, the specificity (proportion of correctly identified post-operation cases) was higher than the sensitivity (proportion of correctly identified pre-operation cases). RF delivered specificity and sensitivity values of $78 \%$ and $70 \%$. In contrast, SVM rendered higher sensitivity. The specificity and sensitivity rates of SVM classifier were $75 \%$ and $80 \%$, respectively.

\section{Discussion and Conclusions}

A two-stage non-parametric artefact removal was adopted in this study mainly because the signals contain artefacts with different probability distributions. The twostage artefact removal was selected empirically.

By considering translational (SCG) and rotational (GCG) precordial vibration patterns, we could moderately discriminate pre- and post-operation states of MI patients who went through PCI.

As shown in Fig. 3, the SCG and GCG signals recorded before the PCI look different from the signals recorded afterwards. According to our observations, identifying such dissimilarities, although dependent on the anatomical location of the atherosclerotic plaque, can be feasible using acceleration and angular velocity signals recorded by smartphones.

Classifying the heart conditions of STEMI patients, us- 

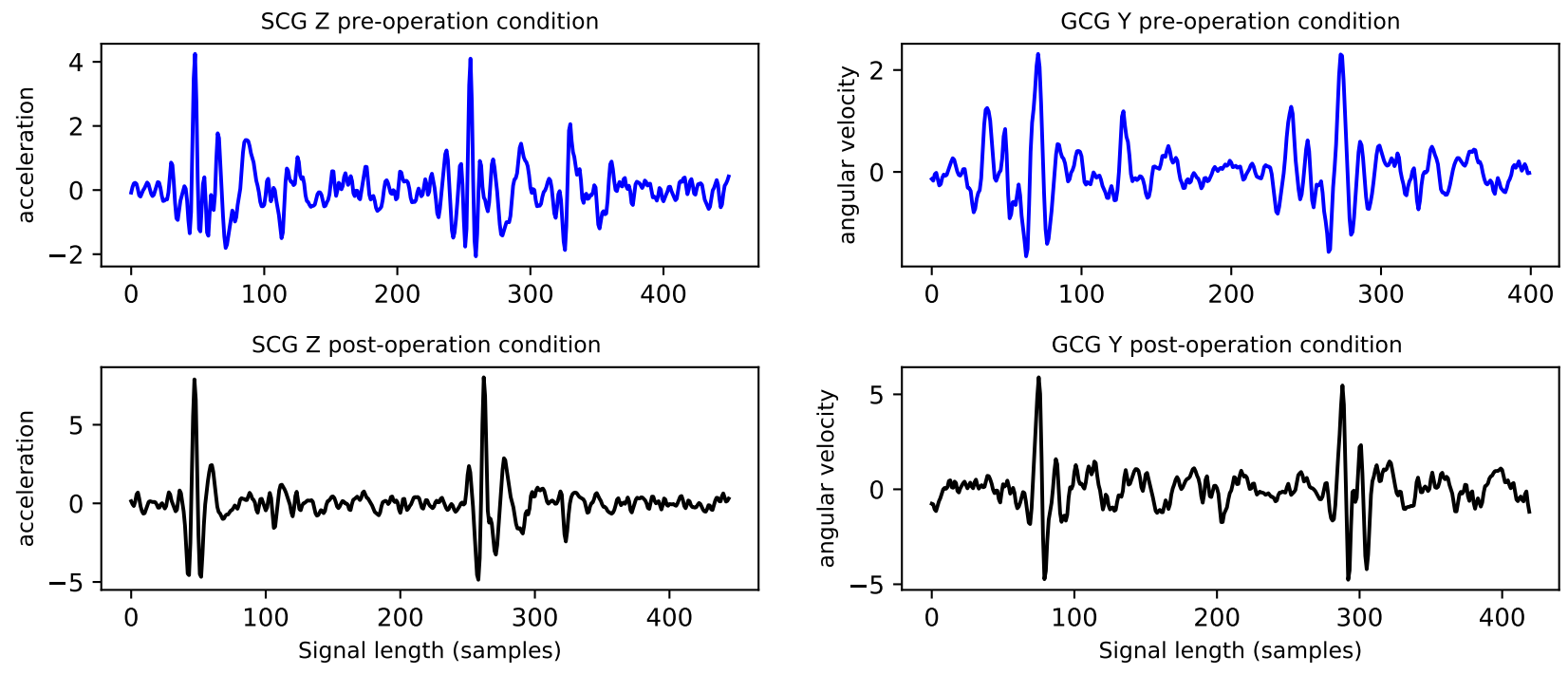

Figure 3. The morphological changes of Z-axis of SCG and Y-axis of GCG for a sample subject. The upper row corresponds to pre-operation while the bottom row corresponds to post-operation.

ing ubiquitous technologies different from the gold standard ECG, may be possible in near future. This study together with our previous contribution by Lahdenoja et al. [8] serve as initial indications of possibility of implementing such a system utilizing only MCG signals.

\section{References}

[1] Myerburg RJ, Kessler KM, Castellanos A. Sudden cardiac death. structure, function, and time-dependence of risk. Circulation 1992;85(1 Suppl):I2-10.

[2] Tung YC, Chang GM, Chang HY, Yu TH. Relationship between early physician follow-up and 30-day readmission after acute myocardial infarction and heart failure. PloS One 2017; 12(1):e0170061.

[3] Ellenius J, Groth T, Lindahl B, Wallentin L. Early assessment of patients with suspected acute myocardial infarction by biochemical monitoring and neural network analysis. Clinical Chemistry 1997;43(10):1919-1925.

[4] Deftereos S, Papoutsidakis N, Giannopoulos G, Kossyvakis C, Lekakis J. Remote monitoring of the cardiac rhythm: where do we stand today? Continuing Cardiology Education 2016;2(4):168-175.

[5] Inan OT, Migeotte PF, Park KS, Etemadi M, Tavakolian K, Casanella R, Zanetti JM, Tank J, Funtova I, Prisk GK, et al. Ballistocardiography and seismocardiography: a review of recent advances. IEEE $\mathbf{J}$ Biomedical and Health Informatics 2015;19(4):1414-1427.

[6] Tadi MJ, Lehtonen E, Saraste A, Tuominen J, Koskinen J, Teräs M, Airaksinen J, Pänkäälä M, Koivisto T. Gyrocardiography: A new non-invasive monitoring method for the assessment of cardiac mechanics and the estimation of hemodynamic variables. Scientific Reports 2017;7(1):6823.
[7] Inan OT, Baran Pouyan M, Javaid AQ, Dowling S, Etemadi M, Dorier A, Heller JA, Bicen AO, Roy S, De Marco T, et al. Novel wearable seismocardiography and machine learning algorithms can assess clinical status of heart failure patients. Circulation Heart Failure 2018;11(1):e004313.

[8] Lahdenoja O, Koivisto T, Tadi MJ, Iftikhar Z, Hurnanen T, Vasankari T, Kiviniemi T, Airaksinen J, Pänkäälä M. A smartphone-only solution for detecting indications of acute myocardial infarction. In Biomedical \& Health Informatics (BHI), 2017 IEEE EMBS International Conference on. IEEE, 2017; 197-200.

[9] Mehrang S, Helander E, Pavel M, Chieh A, Korhonen I. Outlier detection in weight time series of connected scales. In Bioinformatics and Biomedicine (BIBM), 2015 IEEE International Conference on. IEEE, 2015; 1489-1496.

[10] Arora S, Venkataraman V, Donohue S, Biglan KM, Dorsey ER, Little MA. High accuracy discrimination of parkinson's disease participants from healthy controls using smartphones. In Acoustics, Speech and Signal Processing (ICASSP), 2014 IEEE International Conference on. IEEE, 2014; 3641-3644.

[11] Breiman L. Random forests. Machine Learning 2001; 45(1):5-32.

[12] Cortes C, Vapnik V. Support-vector networks. Machine Learning 1995;20(3):273-297.

[13] Oshiro TM, Perez PS, Baranauskas JA. How many trees in a random forest? In MLDM. Springer, 2012; 154-168.

Address for correspondence:

Saeed Mehrang (saeed.mehrang@utu.fi)

Department of Future Technologies

Vesilinnantie 5, 20500 Turku 